\section{Needle-compatible single fiber bundle image guide reflectance endoscope}

\author{
Jiantang Sun, ${ }^{a}$ Chenghao Shu, ${ }^{a}$ Benjamin Appiah, ${ }^{b}$ and \\ Rebekah Drezek ${ }^{a, b, *}$ \\ ${ }^{a}$ Rice University, Department of Bioengineering, 6100 Main \\ Street, Houston, Texas 77005 \\ ${ }^{\mathrm{b}}$ Rice University, Department of Electrical and Computer \\ Engineering, 6100 Main Street, Houston, \\ Texas 77005
}

\begin{abstract}
We developed a miniaturized high-resolution low-cost reflectance-mode fiber microscope (RFM) aimed at optical tissue biopsy applications using a polarized imaging configuration to suppress background noise from specular reflectance. The RFM is equipped with an aircooled light-emitting-diode illumination module and a single $450-\mu \mathrm{m}$ outer-diameter fiber bundle image guide compatible with a 20-gauge needle. The dual illumination and image acquisition nature of the fiber bundle simplifies the system and reduces the total cost. Imaging tests with a United States Air Force resolution target demonstrate a lateral resolution of $\sim 3.5 \mu \mathrm{m}$. The performance of the imaging system is evaluated by examining samples of cells and excised human tissue. Still and video rate images are obtainable in reflectance mode using intrinsic contrast. () 2010 Society of Photo-Optical Instrumentation Engineers. [DOI: 10.1117/1.3465558]
\end{abstract}

Keywords: reflectance fiber endoscope; optical biopsy.

Paper 10220LR received Apr. 26, 2010; revised manuscript received Jun. 11, 2010; accepted for publication Jun. 23, 2010; published online Jul. 29, 2010.

Standard pathology requires removal of cells or tissue through techniques ranging from fine needle aspiration or core needle biopsy to surgical resection. To allow examination of suspect lesions, a variety of endoscopic imaging techniques may be employed, including fiber-scanning confocal microscopy in reflectance $e^{1,2}$ and fluorescence ${ }^{3}$ modes. These techniques use an optical fiber or fiber bundle to view the surface of interior body cavities. Images are created by scanning mechanisms positioned at either the distal or proximal ends of the fiber. These types of systems can capture point-by-point high-resolution images from a large field of view, and the sensitivity of the systems can be very high. ${ }^{4,5}$ Recent advances provide similar high-resolution images using simple, low-cost systems based on coherent fiber bundles with images captured using inexpensive digital cameras. ${ }^{6-8}$ Image resolution is limited by the individual fibers of the fiber bundle. A fluorescentmode fiber endoscope developed by Muldoon et al. achieves a resolution of $\sim 4.4 \mu \mathrm{m}$. ${ }^{6,7}$ We are developing a highresolution $(\sim 3.5 \mu \mathrm{m})$ reflectance-mode endoscope that is flexible, compatible with a 20-gauge needle, and can generate

*Address all correspondence to: Rebekah Drezek, Tel: 713-348-3011; Fax: 713348-5877; E-mail: drezek@rice.edu

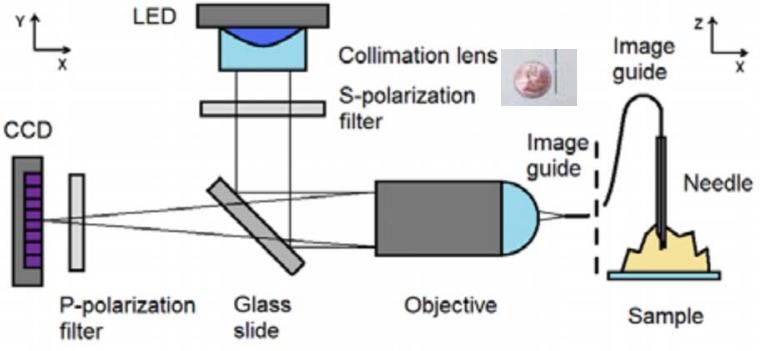

Fig. 1 Schematic of the reflectance fiber imaging system.

still and video rate images using intrinsic contrast. Reflectance-mode endoscopic imaging can potentially complement fluorescence imaging by capturing information not available in fluorescence mode, and by functioning in situations when fluorescent imaging is not practical.

In this work, we present a needle-based, high-resolution reflectance-mode fiber microscope (RFM) developed for optical tissue biopsy applications. The RFM was assembled on a portable $18 \times 24 \times 1 / 2$ in. $^{3}$ aluminum breadboard to facilitate eventual clinical trials. As shown in Fig. 1, an air-cooled lightemitting diode (LED) (Luxeon, Brantford, Ontario, Canada; white LED, model LXHL-NWE8; blue LED, model LXHLNRR8) served as the illumination source to keep the RFM as compact as possible and reduce the system cost. The light from the LED is collimated by an integrated collimating lens and passes through a linear polarizer (Fig. 1), after which the polarized light is reflected by a glass slide positioned at $\sim 45 \mathrm{deg}$ (with respect to the beam trajectory) and projected onto an infinity-corrected $10 \times$ objective lens (Newport, Irvine, California, model L-10×, 0.25 NA). The objective lens and a fiber chuck (Newport, model FPH-DJ) are positioned with a multimode fiber-coupler positioner (Newport, model F-915T). By careful alignment, the illumination light is focused into the incident end of a 450- $\mu \mathrm{m}$ outer-diameter fiber image guide positioned by the fiber chuck (Sumitomo Electric U.S.A., Los Angeles, California, model IGN-037/10, $10^{4}$ fiber elements in a picture area of $333 \mu \mathrm{m}$ diameter, $0.35 \mathrm{NA}){ }^{9}$ Finally, the imaging end of the fiber image guide is inserted into the core of a 20-gauge needle and positioned in contact with the samples. Light scattered from the samples is collected by the same illumination fiber and passes back through the same $10 \times$ objective lens and glass slide described previously (see Fig. 1).

A charge-couple device (CCD) color camera (Edmund Optics, Barrington, New Jersey, model EO-0813C) coupled with a circular polarizer filter (Nikon, $62 \mathrm{~mm}$ ) is used to sample the fiber bundle elements for image acquisition. Because the polarization orientation of the S-polarized illumination light is perpendicular to the plane of incidence with respect to the glass slide (i.e., the XY plane of Fig. 1), a significant portion is reflected toward the incident end of the fiber bundle to be delivered to the imaging end of the fiber bundle. Specular reflectance from the incident end of fiber bundle is also partly reflected away by the glass slide, and the remaining specular reflectance is further suppressed by the circular polarizer in front of the $\mathrm{CCD}$ camera that passes P-polarized light with

1083-3668/2010/15(4)/040502/3/\$25.00 @ 2010 SPIE 


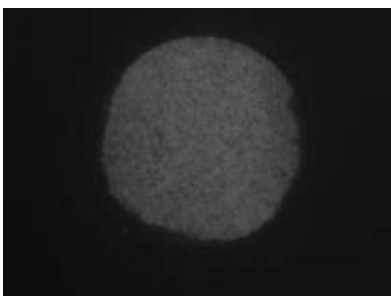

(a)

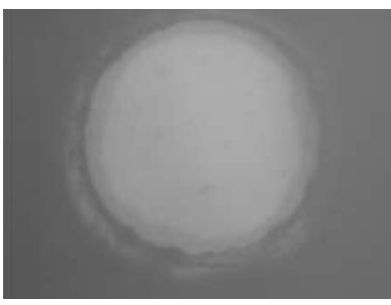

(c)

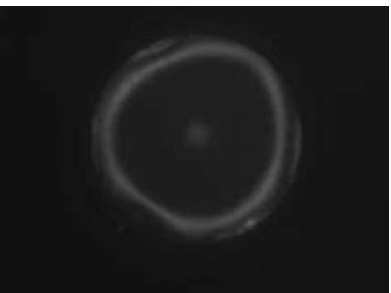

(b)

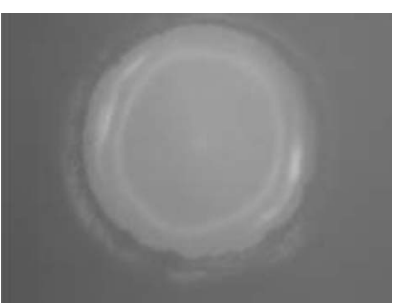

(d)
Fig. 2 Reflectance images of the fiber-bundle tip glass/air interface showing greatly reduced specular background using this RFM system in comparison with the nonpolarized imaging system. Images (a) and (b) were obtained using cross-polarized illumination and imaging filters. For image (a), the fiber tip was in air. For image (b), the fiber tip was covered with a drop of water. Images (c) and (d) were obtained without polarized illumination and imaging filters. Image (c) is with the fiber tip in air, whereas image (d) is with the fiber tip covered with a drop of water.

respect to the plane of incidence. Rays that result from multiple scattering events changing their polarization to the parallel direction can be detected by the CCD camera (e.g., light scattered from the sample). Thus, noise due to specular reflectance from the incident end of the fiber bundle is greatly suppressed, and clearer images are collected.

Figure 2(a) shows a reflectance image of the fiber bundle imaging end when the needle microscope is positioned in air. Light from the white LED that is reflected from the glass-air interface at the imaging end of the fiber bundle is successfully detected by the CCD camera, and individual fibers of the fiber bundle are resolved [Fig. 2(a)]. With the imaging needle of the RFM immersed into a vial of water or phosphate-buffered saline (PBS) solution, the RFM field of view is almost completely dark, because the close match between the refractive indices of water and the glass fiber result in dramatically reduced internal reflection from the imaging end of the fiber bundle.

Figure 2(b) is obtained with a drop of water covering the tip of the imaging fiber bundle, which mimics the immersion of the fiber tip in water, to provide clear image features. Much less internal reflection occurs from the imaging area covered by water [Fig. 2(b)] compared with the same area when it is exposed to air. Figures 2(a) and 2(b) were captured with the same imaging configurations and the index-matching effect is clear. When used in biological or clinical environments, the fiber bundle tip is always in contact with body fluids or tissue. Thus, dipping the fiber bundle tip into water roughly approximates the conditions of realistic imaging applications. In liquid, the reduced internal reflectance leads to a dark field of view and a decrease in background enabling biological imaging. Light scattered by biological structures can be clearly distinguished and cellular images can be obtained. In contrast to the polarized reflectance-mode images in Figs. 2(a) and

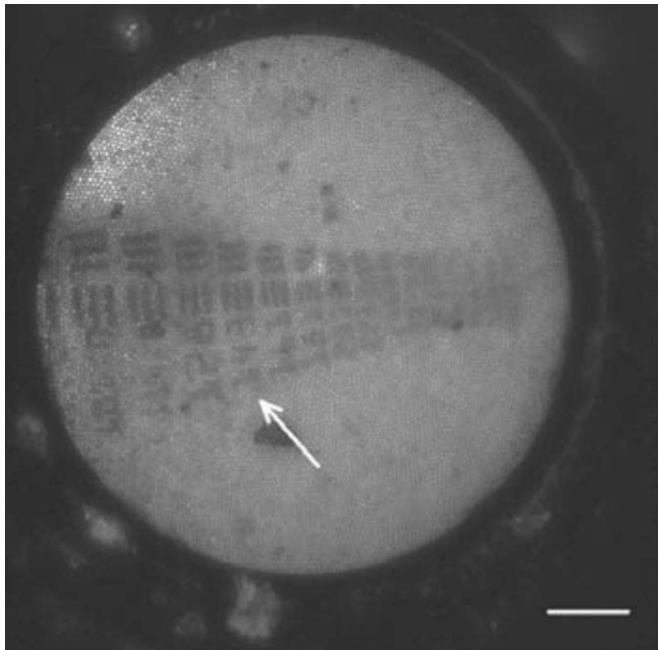

(a)

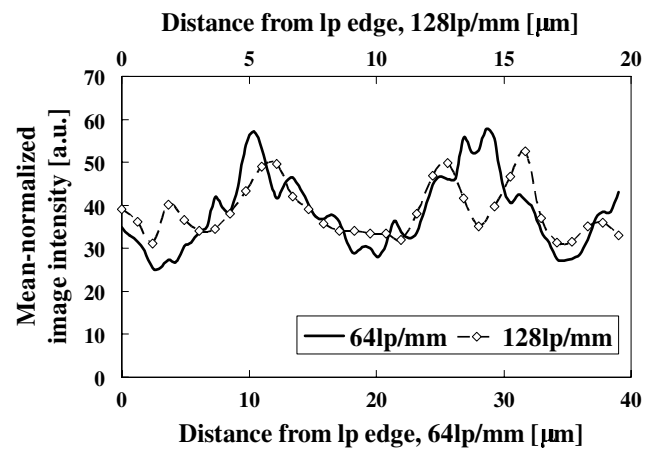

(b)

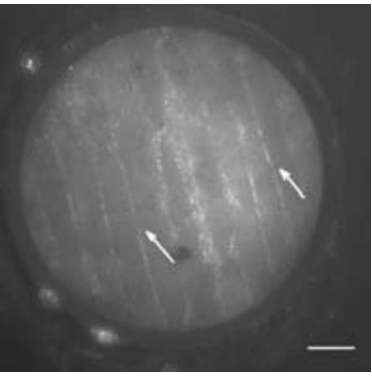

(c)

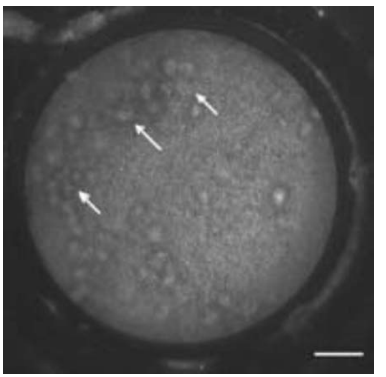

(d)
Fig. 3 Processed endoscopic imaging results of (a) USAF resolution target showing the resolution of $\sim 143 \mathrm{lp} / \mathrm{mm}$, acquired with white LED illumination, (b) cross sections of two resolution line-pair sets showing both the line-pair intensity variation and variation due to probe fibers (3.3 $\mu \mathrm{m}$ diam), (c) onion skin cells (see also Video 1), where arrows show the resolved cell walls acquired with white LED illumination, and (d) cultured SKBR3 cancer cells (see also Video 2), where the arrows indicate resolved single cells acquired with blue LED illumination. Scale bar is $50 \mu \mathrm{m}$. (Video 1, QuickTime, $754 \mathrm{~KB}$ [URL: http://dx.doi.org/10.1117/1.3465558.1]; Video 2, QuickTime, 977 KB [URL: http://dx.doi.org/10.1117/1.3465558.2].)

2(b), images shown in Figs. 2(c) and 2(d) were captured with a reduced exposure time under the same imaging conditions, but without the illumination and collection polarizers. The specular reflectance from the optical components makes it impossible to obtain useful information from Figs. 2(c) and 2(d).

A white LED was used to acquire the images and plots 


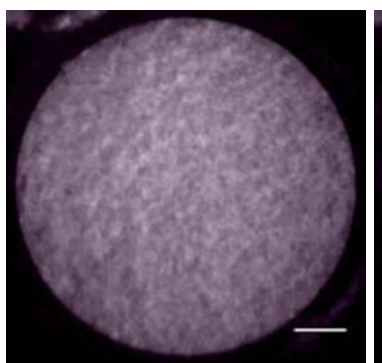

(a)

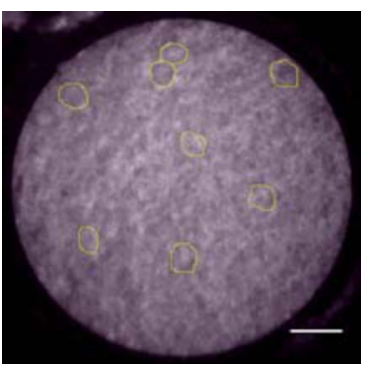

(b)

Fig. 4 Endoscopic images of excised oral cancer tissue including (a) processed image showing cellular structures (see also Video 3, where images move in and out of focus as the imaging tip moves), and (b) processed images with drawn lines superimposed to indicate the estimated cell boundaries. Scale bar is $50 \mu \mathrm{m}$. (Video 3, QuickTime, 2.12 MB [URL: http://dx.doi.org/10.1117/1.3465558.3].)

shown in Figs. 3(a)-3(c) and Video 1. Figure 3(a) shows an imaging resolution of $\sim 3.5 \mu \mathrm{m}(\sim 143$ line pairs/mm $)$ obtained upon imaging the United States Air Force resolution target. Figure 3(b) shows that contrast decreases slightly from low $(64 \mathrm{lp} / \mathrm{mm})$ to high $(128 \mathrm{lp} / \mathrm{mm})$ resolution, but it also reveals high-frequency components due to the probe fibers (3.3 $\mu \mathrm{m}$ diam). Figure 3(c) and Video 1 were obtained by using the same white LED-illuminated RFM to image skin tissue from a green onion. The cell walls of the rectangular green onion skin cells ${ }^{10}$ are clearly resolved in both the still image and the video. Note that water was used in all the tests to provide refractive index matching.

The LED light source is cost effective and easy to change. An air-cooled blue LED was used to image cultured SKBR3 human carcinoma cells. Before imaging, SKBR3 carcinoma cells were cultured in a $6-\mathrm{mm}$ plate at $37{ }^{\circ} \mathrm{C}$ for 2 days. The fiber bundle tip was immersed into the culture medium through a needle and focused onto a group of cells attached to the culture plate. As shown in Fig. 3(d), the cells can be visualized with the RFM using blue-LED illumination. An endoscopic video clip of the SKBR3 cancer cells is provided in Video 2. The same RFM was also used to image excised human oral cancer tissue obtained from the Cooperative $\mathrm{Hu}-$ man Tissue Network (CHTN, Rockville, Maryland). The tissue was placed in a culture plate with PBS, which was then placed on a 2-D translation stage. The needle and fiber bundle tip were inserted into the tissue sample, and cellular structures were visualized as shown in Fig. 4 and Video 3. In Video 3, cellular images move in and out of focus as the imaging tip moves closer to or further away from the sample. The same RFM was used to image the reflectance from a dust particle in liquid solution to demonstrate single pixel fiber resolution, as shown in Video 4.

In this work, we describe a high-resolution RFM developed for optical tissue biopsy applications. The resolution of this fiber microscope is limited by the element fiber size of the imaging fiber bundle. For the specific fiber bundle image guide presented, the resolution is $\sim 3.5 \mu \mathrm{m}$. The reported RFM system uses the same fiber bundle guide for both illumination and imaging. A pair of cross-polarized filters is used in addition to the fiber bundle guide to achieve highresolution reflectance-mode microscopic imaging. The fiber bundle guide is $450 \mu \mathrm{m}$ in diameter, making it compatible

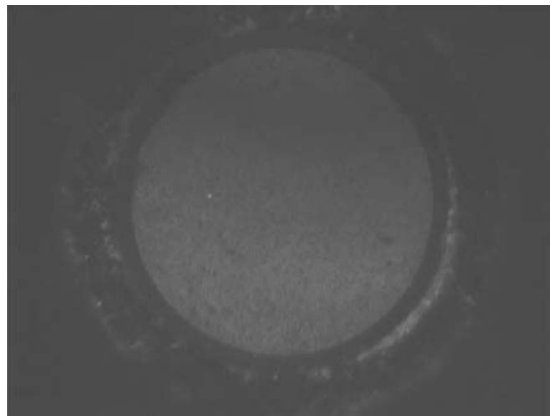

Video 4 Reflectance endoscopic video of a dust particle in liquid solution showing single pixel fiber resolution. (QuickTime, $950 \mathrm{~KB}$ ). [URL: http://dx.doi.org/10.1117/1.3465558.4].

with current clinical needle tissue biopsy systems. The RFM system can be used without external agents when there is sufficient endogenous imaging contrast. Moreover, with simple filter changes, the RFM system can readily be converted to a fluorescence-mode needle biopsy imaging system similar to that developed by Muldoon et al. ${ }^{6}$ Future work will assess whether performance can be improved through the use of simple chemical agents already in routine clinical use.

\section{Acknowledgments}

The authors gratefully acknowledge support from the Department of Defense Congressionally Directed Breast Cancer Research Program Era of Hope Scholar Award (W81XWH-071-0428). The NCI Cooperative Human Tissue Network $(\mathrm{CHTN})$ provided specimens used in this project.

\section{References}

1. C. Liang, M. Descour, K. B. Sun, and R. Richards-Kortum, "Fiber confocal reflectance microscope (FCRM) for in-vivo imaging," Opt. Express 9, 821-830 (2001).

2. K. C. Maitland, A. M. Gillenwater, M. D. Williams, A. K. El-Naggar, M. R. Descour, and R. R. Richards-Kortum, "In vivo imaging of oral neoplasia using a miniaturized fiber optic confocal reflectance microscope," Oral Oncol. 44, 1059-1066 (2008).

3. F. Jean, G. Bourg-Heckly, and B. Viellerobe, "Fibered confocal spectroscopy and multicolor imaging system for in vivo fluorescence analysis," Opt. Express 15, 4008-4017 (2007).

4. V. Dubaj, A. Mazzolini, A. Wood, and M. Harris, "Optic fiber bundle contact imaging probe employing a laser scanning confocal microscope," J. Microsc. 207, 108-117 (2002).

5. R. Kiesslich, M. Goetz, M. Vieth, P. R. Galle, and M. F. Neurath, "Technology Insight: confocal laser endoscopy for in vivo diagnosis of colorectal cancer," Nat. Clin. Pract. Oncol. 4, 480-490 (2007).

6. T. J. Muldoon, M. C. Pierce, D. L. Nida, M. D. Williams, A. Gillenwater, and R. Richards-Kortum, "Subcellular-resolution molecular imaging within living tissue by fiber microendoscopy," Opt. Express 15, 16413-16423 (2007).

7. T. J. Muldoon, S. Anandasabapathy, D. Maru, and R. RichardsKortum, "High-resolution imaging in Barrett's esophagus: a novel, low-cost endoscopic microscope," Gastrointest. Endosc. 68, 737-744 (2008).

8. T. J. Muldoon, N. Thekkek, D. Roblyer, D. Maru, N. Harpaz, J. Potack, S. Anandasabapathy, and R. Richards-Kortum, "Evaluation of quantitative image analysis criteria for the high-resolution microendoscopic detection of neoplasia in Barrett's esophagus," $J$. Biomed. Opt. 15, 026027 (2010).

9. Sumitomo Electric U.S.A., Image Guide Technical Document 〈http:// www.sumitomoelectricusa.com/scripts/products/ofig/image.cfm $\rangle$ (2010).

10. A. G. von Arnim, X. W. Deng, and M. G. Stacey, "Cloning vectors for the expression of green fluorescent protein fusion proteins in transgenic plants," Gene 221, 35-43 (1998). 